\title{
Thrombectomy in patients with tandem stenoses
}

\author{
Gerhard Schroth $^{1} \cdot$ Rüdiger von Kummer ${ }^{2}$
}

Received: 24 March 2015 / Accepted: 26 March 2015 /Published online: 10 April 2015

(C) Springer-Verlag Berlin Heidelberg 2015

In this issue, Lockau et al. retrospectively analyze different approaches for endovascular recanalization of tandem occlusions in acute stroke [1]. Starting with stent angioplasty of the carotid occlusion as the first step (stent-first group), they reversed the order of their techniques and performed intracranial thrombectomy prior to stenting of the carotid occlusion (thrombectomy-first group, the so-called upside-down approach). They found advantages and disadvantages with both techniques; however, time of intervention was shorter for the thrombectomy-first group. A higher percentage of thrombectomy-first patients had favorable outcomes after 3 months but the difference was not statistically significant. As discussed in this paper, one of the reasons may be that the thrombectomy-first technique allows mobilization and removal of the intracranial thrombus during flow arrest, whereas the thrombus becomes more compressed due to increased perfusion pressure following stenting of the carotid artery.

This is an article from experts for experts that seek to bring researchers and clinicians together and thereby to improve our approach towards treatment of acute internal carotid artery (ICA) occlusion. This goal is equally or even more challenging than other procedures being developed in the emerging field of interventional neuroradiology. Like embolization of an AVM or coiling of an aneurysm, recanalization in acute stroke is an image-guided endovascular microneurosurgical operation

This comment refers to the article available at: http://dx.doi.org/10.1007/ s00234-014-1465-5.

Rüdiger von Kummer

Ruediger.vonKummer@uniklinikum-dresden.de

1 Institute of Neuroradiology, Inselspital, University of Bern, Bern, Switzerland

2 Institute of Diagnostic and Interventional Neuroradiology, Universitätsklinikum Carl Gustav Carus - Dresden, Fetscherstr. 74, Haus 59, 01307 Dresden, Germany that should be navigated using a biplane or 3D roadmap with a spatial resolution of 150 microns and with a time resolution below $100 \mathrm{~ms}$. For treatment of stroke, however, we must navigate our micro-instruments across the occlusion blindly, without a precise radar picture of our roadmap. Knowledge of the neurovascular anatomy and extensive experience with the whole range of neurointerventional instruments and techniques are preconditions for success.

Ischemic strokes resulting from occlusion of the ICA are often severe and have a poor prognosis. Predictors of improved outcome are good intracranial collaterals and recanalization. Intravenous thrombolysis in patients with tandem stenoses achieves low rates of recanalization-between 0 and $20 \%$-which can be increased to more than $80 \%$ by endovascular treatment.

A systematic review of 28 studies found significantly higher rates of favorable outcome in the endovascular group (33.6 \% of 584 patients with favorable outcome) as compared to the iv thrombolysis group ( $24.9 \%$ of 385 patients with favorable outcome; $p=0.0004$ ) [2]. A recent literature review of 32 studies dealing with endovascular treatment of 1107 patients with acute ischemic stroke and ICA occlusion confirmed these findings [3]. Ten of these studies included patients with tandem occlusions, with an overall favorable clinical outcome in $43 \%$; however, the mortality rate was $45 \%$, and $16 \%$ of patients had symptomatic intracranial hemorrhages. Combined analysis of two studies of patients with tandem occlusions showed a significantly lower death rate for the group treated with intra-arterial thrombolysis and a lower rate of recanalization of the extracranial ICA occlusion (61\% for IA thrombolysis versus $99 \%$ with stenting), whereas the rate of recanalization of intracranial occlusion did not differ significantly (60 versus $78 \%$ ) [3].

The current situation regarding thrombolysis treatment is reminiscent of the situation of endovascular treatment of stroke in general, where - fortunately - after a promising start followed by a period of prospective and randomized confusion, the fog is starting to lift. 
Following the pioneering work of Zeumer and Hacke, experienced interventional neuroradiologists planned and performed the PROACT II study [4]. This study evaluated an inexpensive and relatively safe procedure of local intraarterial thrombolysis in MCA occlusions. Angiographically confirmed recanalization was achieved in $66 \%$ and an increase in good clinical outcomes from $25 \%$ in the control group to $40 \%$ after IA treatment, which is surprisingly high, considering the initial NIHSS of 18 . We have used this technique since 1992, and since 2004, it was used in combination with the aspiration technique after the 5-French aspiration catheter (Vasco-Asp; BALT) was CE-certified. We changed to stent-retriever technology after confirmation by in vitro and animal experiments that this technique enables clot removal en bloc in cases where mobilization and removal of the thrombus is performed under flow arrest.

On the other hand, commercially driven thrombus fragmenting techniques and instruments have been introduced, with a subsequent dramatic decline of favorable clinical outcome. Nevertheless, these techniques and instruments were used primarily or exclusively in the unhappy triad, published in New England Journal of Medicine last year [5-7]. The SWIFT and TREVO 2 trials confirmed the superiority of stent-retrievers over the MERCI retriever with regard to thrombectomy, although clinical outcome was similar in the compared treatment groups. Distal protection by a balloonmounted guiding catheter and/or distal access catheter was not mandatory $[8,9]$. MR CLEAN proved the efficacy of mechanical thrombectomy in a patient group with very poor prognosis after standard therapy [10]. Protection against iatrogenic emboli was again not required by the study protocol and occurred in $8.5 \%$. Consequently, good clinical outcome could only be achieved in $33 \%$ of patients.

The essential step forward was achieved by using stentretriever technology in combination with proximal protection by a balloon-mounted guiding catheter and/or distal protection using a high volume distal access catheter and simultaneous aspiration during retrieval of the stent. Good clinical outcome (mRS 0-2) was increased by more than $20 \%$ compared to controls and by more than $50 \%$ compared to recent trials [11-13].

As noted above, iatrogenic emboli in primary non-affected arteries were detected via angiography in $8.5 \%$ of patients in the MR CLEAN trial. Using susceptibility-weighted imaging (SWI) for quality control before and after mechanical thrombectomy, we detected a large number of distal iatrogenic emboli in patients rated angiographically as TICI 3 recanalization. Angio-control at the end of the procedure is of course mandatory, although we are not sure whether TICI classification is sensitive enough to measure the quality of the technique or detects only the tip of an iceberg. Nevertheless, thromboembolic occlusion of primary non-affected arteries that are serving as collaterals is a severe complication in elective interventional neuroradiology and should never be tolerated in this setting. In stroke, however, the resulting clinical consequences may be neglected and attributed to progression of the primary stroke.

The publication of Lockau et al. [1] in this issue takes us back to essential questions. They observed that changing the procedure may improve clinical outcome. The retrospective exploration of data is, however, hypothesis generating only. A prospective controlled trial with pre-specified endpoints that take into account all potential confounders is necessary to definitively establish whether intracranial recanalization is safer and more effective with the ICA still occluded. Stenting the ICA may allow safer placement of the guiding catheter. Normalized blood flow through the ICA is a precondition for assessing the flow through the recanalized intracranial artery. Local contrast injections do not help to prove normalized blood flow. It might not be necessary in each case to stent the carotid artery. Dottering the stenosis with an 8-French catheter or PTA alone may be sufficient to keep this large vessel open if the flow is high enough following thrombectomy of the distal thrombus. Recanalization of the MCA occlusion is the primary goal of treatment in tandem stenosis. If the result remains stable, which can easily be controlled with bedside Doppler sonography, double aggregation can be avoided. This possibility may be another argument in favor of an "upside-down" approach to recanalization of tandem occlusion-i.e., with distal thrombectomy as the first step. New developments of stents with flow-diverting effects may prevent migration of vulnerable plaque material into the stent lumen, thereby diminishing the risk of acute in-stent thrombosis. Last but not least, online biplane or 3D roadmaps created in the neuroangio suite, using the late phases of CTA, MRA, or flat panel rotation angiography will enable matching of the virtual anatomy of the vessels behind the site of occlusion in real time in patients with sufficient collateral flow. In combination with thrombus imaging, we can precisely guide our micro-instruments safely beyond the occlusion and into the branch of the MCA, where the thrombus extends.

A lack of attention or knowledge about the individual vascular pathology, propagation of technical approaches that are not supported by clinical trial results, and neglect of the widespread experience of experts may lead to the illusion that new stent-retrievers or the 10-year-old aspiration technology are "no brainers" that can be applied by anybody, without knowledge of basic neuroscience and solid training in endovascular, image-guided micro-neurosurgery.

Ethical standards and patient consent We declare that this Invited Editorial does not contain original clinical studies or patient data.

Conflict of interest GS offers services and consults for Industry; however, the funds received are exclusively paid to the scientific fond of the University Hospital Bern, Institute for Neuroradiology. RvK has received honoraria for services on steering committees, safety committees, and 
adjudication committees from Covidien, Penumbra, Boehringer Ingelheim, Lundbeck, Brainsgate and Synarc.

\section{References}

1. Lockau H, Liebig T, Henning T, Neuschmelting V, Stetefeld H, Kabbasch C et al (2015) Mechanical thrombectomy in tandem occlusion: procedural considerations and clinical results. Neuroradiology 215:xxx-xxx. doi:10.1007/s00234-014-1465-5

2. Mokin M, Kass-Hout T, Kass-Hout O, Dumont TM, Kan P, Snyder KV et al (2012) Intravenous thrombolysis and endovascular therapy for acute ischemic stroke with internal carotid artery occlusion: a systematic review of clinical outcomes. Stroke 43:2362-2368. doi: 10.1161/STROKEAHA.112.655621

3. Kappelhof M, Marquering H, Berkhemer O, CBLM M (2015) Intra-arterial treatment of patients with acute ischemic stroke and internal carotid occlusion: a literature review. J Neurointerv Surg 7: 8-15. doi:10.1136/neurintsurg-2013-011004

4. Furlan A, Higashida R, Wechsler L, Gent M, Rowley H, Kase C et al (1999) Intra-arterial prourokinase for acute ischemic stroke. The PROACT II study: a randomized controlled trial. Prolyse in acute cerebral thromboembolism. JAMA 282:2003-2011

5. Broderick JP, Palesch YY, Demchuk AM, Yeatts SD, Khatri P, Hill MD et al (2013) Interventional management of stroke IIII. Endovascular therapy after intravenous t-PA versus t-PA alone for stroke. New Engl J Med 368:893-903. doi:10.1056/ NEJMoa1214300

6. Ciccone A, Valvassori L, Investigators SE (2013) Endovascular treatment for acute ischemic stroke. New Engl J Med 368:24332434
7. Kidwell CS, Jahan R, Gornbein J, Alger JR, Nenov V, Ajani Z et al (2013) Investigators MR. A trial of imaging selection and endovascular treatment for ischemic stroke. The New Engl J Med 368:914-923. doi:10.1056/NEJMoa1212793

8. Saver JL, Jahan R, Levy EI, Jovin TG, Baxter B, Nogueira R et al (2014) SOLITAIRE with the intention for thrombectomy (SWIFT) trial: design of a randomized, controlled, multicenter study comparing the SOLITAIRE flow restoration device and the MERCI retriever in acute ischaemic stroke. Int J Stroke 9:658-668. doi:10. 1111/j.1747-4949.2012.00856.x

9. Nogueira RG, Lutsep HL, Gupta R, Jovin TG, Albers GW, Walker GA et al (2012) Trevo versus Merci retrievers for thrombectomy revascularisation of large vessel occlusions in acute ischaemic stroke (TREVO 2): a randomised trial. Lancet 380:1231-1240. doi:10.1016/S0140-6736(12)61299-9

10. Berkhemer OA, Fransen PS, Beumer D, van den Berg LA, Lingsma HF, Yoo AJ et al (2015) A randomized trial of intraarterial treatment for acute ischemic stroke. New Engl J Med 372:11-20. doi:10. 1056/NEJMoa1411587

11. Campbell BC, Mitchell PJ, Kleinig TJ, Dewey HM, Churilov L, Yassi $\mathrm{N}$ et al (2015) Endovascular therapy for ischemic stroke with perfusion-imaging selection. New Engl J Med 372:1009-1018. doi: 10.1056/NEJMoa1414792

12. Goyal M, Demchuk AM, Menon BK, Eesa M, Rempel JL, Thornton $\mathrm{J}$ et al (2015) Randomized assessment of rapid endovascular treatment of ischemic stroke. New Engl J Med 372: 1019-1030. doi:10.1056/NEJMoa1414905

13. Pereira VM, Gralla J, Davalos A, Bonafe A, Castano C, Chapot R et al (2013) Prospective, multicenter, single-arm study of mechanical thrombectomy using Solitaire Flow Restoration in acute ischemic stroke. Stroke 44:2802-2807. doi:10.1161/STROKEAHA. 113.001232 